\title{
Isolation and Characterization of Densonucleosis Virus from Aedes aegypti Mosquitoes and Its Distribution in India
}

\author{
A. Sivaram P.V. Barde S.R.P. Kumar P. Yadav M.D. Gokhale A. Basu \\ D.T. Mourya \\ Microbial Containment Complex, National Institute of Virology, Pune, India
}

\section{Key Words}

Densonucleosis virus • Mosquitoes · Aedes aegypti .

Phylogenetic analysis

\begin{abstract}
Objectives: Mosquito densonucleosis viruses (DNVs) are known to persistently infect the insect cell line and mosquito population in nature, causing mortality in mosquitoes. Here we report the isolation and characterization of a DNV from Aedes aegypti and its distribution among different $A e$. aegypti populations from India. Methods: We screened Ae. aegypti mosquito populations from different states of India by PCR. Virus isolation and purification was performed using a cesium chloride gradient from a positive mosquito colony. Characterization of this isolate was carried out by electron microscopy, Western blot and sequencing. Results: Electron microscopy showed the presence of parvovirus-like particles, and Western blot showed the presence of 2 viral proteins of 40 and $41 \mathrm{kDa}$. A total of 3,776 bases of genome were sequenced, which included a 3'UTR of 128 bases, a coding region of 3,507 bases and a 5'UTR of 141 bases. Three open reading frames (ORFs) were identified and characterized. The NIVDNV genome showed $95 \%$ similarity with Culex pipiens pallens DNV and 93\% similarity with Ae. aegypti DNV.
\end{abstract}

Conclusion: Phylogenetic analysis of all 3 ORFs showed that this new isolate falls in the lineage of Brevidensovirus along with other mosquito DNVs.

Copyright $\odot 2009$ S. Karger AG, Basel

\section{Introduction}

Densonucleosis viruses (DNVs) are the etiological agents of the insect disease known as densonucleosis [1] and belong to the family Parvoviridae. They infect and replicate only within insects. Densonucleosis disease leads to either death or the loss of vital functions in all stages of infected organisms. Densonucleosis was first discovered in the caterpillar Galleria mellonella, resulting in the isolation of G. mellonella DNV. Since then, DNVs have been isolated from a wide range of economically or medically important insects including the orders Lepidoptera, Diptera, Orthoptera and Dictyoptera. The first mosquito densovirus (MDV) was isolated from an infected laboratory colony of Aedes aegypti and was named Ae. aegypti DNV (AeDNV) [2]. DNVs have also been isolated from other mosquitoes such as Ae. albopictus [3], Culex pipiens [4], Toxorhynchites splendens [5] and Anopheles minimus [6] as well as from mosquito cell lines [7].

\section{KARGER}

Fax +4161306 1234 E-Mail karger@karger.ch www.karger.com
(C) 2009 S. Karger AG, Basel

0300-5526/09/0521-0001\$26.00/0

Accessible online at:

www.karger.com/int
D.T. Mourya

Microbial Containment Complex

Sus Road

Pashan, Pune 411021 (India)

Tel./Fax +91 202587 0640, E-Mail mouryadt@icmr.org.in 
DNVs are single-stranded linear DNA viruses belonging to the genus Brevidensovirus of the subfamily Densovirinae. Each DNV virion packages either a plus or a minus DNA strand. The virion appears as an icosahedral, nonenveloped particle with a diameter of $18-26 \mathrm{~nm}$. Sequence analysis and comparison showed that the nucleotide and amino acid sequences of Ae. albopictus C6/36 cell DNV (C6/36DNV) are highly homologous to the AeDNV genome, but the genomic organization and some repeating sequences of C6/36DNV are more closely related to those of Ae. albopictus DNV (AalDNV) [8].

Both AeDNV and AalDNV are highly pathogenic for mosquito cell lines and all mosquito tissues, killing up to $100 \%$ of early-stage larvae $[9,10]$. However, the rate of mortality decreases if the virus infects during the later stages of larval development, as the larvae can survive infection and develop into adults. Infected mosquitoes can also transmit the virus vertically to the next generation $[9,11,12]$.

DNVs have recently gained a lot of scientific interest because of their potential as a system for delivering foreign genes to mosquitoes and for the biological control of the activity of mosquitoes. It has also been reported that the infection of Aedes mosquitoes with DNV greatly reduces their susceptibility to dengue virus $[13,14]$. With the growing threat of emerging viruses, especially arboviruses, the role played by such endogenously infected viruses in vector dynamics and transmission constitutes a need-of-the-hour research question.

During the recent outbreak of chikungunya virus in India, mosquitoes were received by our laboratory from various states in India for the detection of arboviruses. Further investigations showed the presence of DNV in certain populations. The present communication reports the first isolation and molecular characterization of a $\mathrm{DNV}$ from India and its distribution among various $A e$. aegypti mosquito populations across different states.

\section{Materials and Methods}

Development of the DNV-Infected Colony of Ae. aegypti

The Ae. aegypti mosquito colony used for these experiments was from an insectary maintained at this institute for the last 25 years. The mosquitoes were maintained at $28 \pm 2^{\circ}$ and a relative humidity of $70-80 \%$. The larvae were fed on yeast tablets and dog biscuits mixed in the proportion of 70 and $30 \%$, respectively. The adults were reared in cages and were fed on $10 \%$ glucose. Females were fed with chicken blood meal every third day to obtain eggs.

Ae. aegypti mosquitoes caught in the field were screened for DNV infection by polymerase chain reaction (PCR) [14]. The mosquitoes found to be infected with DNV were triturated in phosphate-buffered saline (PBS) and centrifuged at 5,000 rpm to remove the cell debris, and the supernatant was added to the uninfected larval pan containing the first-instar Ae. aegypti larvae. When these larvae reached the fourth instar, they were tested for DNV infection using the protocol described by Wei et al. [14]. This process was repeated to generate the DNV-infected mosquito colony.

\section{Isolation and Purification of DNV}

Approximately 10,000 fourth-instar larvae from the infected colony were triturated in $150 \mathrm{ml}$ of PBS using a grinder (Fisher Scientific). The slurry was centrifuged at $10,000 \mathrm{rpm}$ for $30 \mathrm{~min}$ to remove the cell debris. The supernatant was divided into $1-\mathrm{ml}$ aliquots and stored at $-80^{\circ}$. This was used as the viral stock throughout the study. Four milliliters of the virus stock was filtered using $0.22-\mu \mathrm{m}$ filters. It was then centrifuged at $25,000 \mathrm{rpm}$ for $2 \mathrm{~h}$ at $4^{\circ}$ in an ultracentrifuge on a cushion of $30 \%$ glycerol in NTE buffer ( $\mathrm{NaCl} 100 \mathrm{~mm}$, Tris HCl 10 mM, EDTA 4 mM, pH 7.4). The pellet obtained was dissolved in $1 \mathrm{ml}$ of PBS and was centrifuged at $40,000 \mathrm{rpm}$ for $4 \mathrm{~h}$ at $4^{\circ}$ on a $30-60 \%$ discontinuous cesium chloride $(\mathrm{CsCl})$ gradient. Each fraction was separated and analyzed by transmission electron microscopy, PCR and sodium dodecyl sulfate polyacrylamide gels (SDS-PAGE).

\section{Raising Immune Sera and Purification of IgG}

The purified fraction of the DNV was used to immunize adult Swiss albino mice via the intraperitoneal route. Three booster doses were given at intervals of 1 week. One week after the final dose, blood from immunized mice was collected by orbital bleeding and immune sera were separated. The immune sera were adsorbed against fresh Ae. aegypti larvae from the normal colony to remove the antibodies raised against the mosquito proteins. The adsorption was performed by incubating $500 \mu \mathrm{l}$ of immune sera with $500 \mu \mathrm{l}$ of larval suspension for $2 \mathrm{~h}$ at room temperature in a rocker. This was then centrifuged at 10,000 rpm for $20 \mathrm{~min}$. The pellet was discarded and the supernatant was used to purify IgG with the Protein G column (Sigma-Aldrich, St. Louis, Mo., USA) following the manufacturer's instructions.

\section{SDS-PAGE and Western Blot}

The different fractions of gradient purification and the immunoprecipitate were analyzed by SDS-PAGE using $4 \%$ stacking and $12 \%$ resolving acrylamide gels, with a constant voltage of $100 \mathrm{~V}$. The gels were stained by the silver staining method [15]. The gradient centrifuge fraction that showed the bands for the suspected proteins in SDS-PAGE was used for immunoprecipitation with the purified IgG. For immunoprecipitation, $50 \mu \mathrm{l}$ of a $10^{-1}$ dilution of IgG was incubated with $50 \mu$ l of purified virus (from the gradient centrifuge fraction) on a rocker for $2 \mathrm{~h}$ at $4^{\circ}$. This was then centrifuged at $14,000 \mathrm{rpm}$ for $30 \mathrm{~min}$. The supernatant was stored separately and the pellet was suspended in $30 \mu \mathrm{l}$ of NTE buffer. The proteins were transferred to a nitrocellulose membrane. The nitrocellulose membrane was blocked using blocking buffer composed of $4 \%$ bovine serum albumin and $0.05 \%$ Tween20 in PBS. The purified IgG raised against the DNV was diluted in PBS (1:200) and used to probe the virus. The reaction was determined using antimouse antibodies tagged with alkaline phosphatase (1:25,000) and 5-bromo-4-chloro-3-indonyl phosphate as substrate (Sigma). 
Table 1. Genomic location of the primers and their sequences

\begin{tabular}{clcl}
\hline No. & Primer name & $\begin{array}{c}\text { Genomic } \\
\text { location }\end{array}$ & Sequence \\
\hline 1 & DNV 32 F & $32-55$ & TTTCGTGATACGGATACTGTAGAT \\
2 & DNV 232 F & $232-255$ & CTTAATTCCACTACCACATGGTC \\
3 & DNV 570 F & $570-592$ & GGTGACCTCTACCCACAATTAC \\
4 & DNV 1462 F & $1462-1481$ & ACAGAAGCGATGGATACG \\
5 & DNV 1745 F & $1745-1768$ & ACGCATCGAAAGAACACGTATAC \\
6 & DNV 2688 F & $2688-2713$ & GACCTAATATGAGCGAAATGGTACC \\
7 & DNV 3401 F & $3401-3428$ & ACATCATATCCTAGAATGCATATGGCA \\
8 & DNV 302 R & $302-322$ & AATGTTCAACGCAGACTGAA \\
9 & DNV 648 R & $648-670$ & GTACAAGTATTTCCCAGCTTCC \\
10 & DNV 805 R & $805-826$ & ATCTTTGGACAGCGTCTCGTA \\
11 & DNV 2238 R & $2238-2256$ & TGTGATCCACGTTGGTGT \\
12 & DNV 3239 R & $3239-3258$ & CTGCAGCGCTAATGTCTGT \\
13 & DNV 3754 R & $3754-3771$ & AGGTGGAATACGGAGGTG \\
\hline
\end{tabular}

Transmisson Electron Microscopy

$\mathrm{CsCl}$ gradient-purified virus was examined by negative staining in a transmission electron microscope. Briefly, $100 \mu \mathrm{l}$ of the fraction was adsorbed on a carbon-coated Formvar support on 400 mesh copper grids (Polysciences, Sunnyvale, Calif., USA) for $20 \mathrm{~min}$, excess fluid was removed by capillary blotting and the grids were negatively stained with $1 \%$ phosphotungstic acid. Specimens were then examined in a transmission electron microscope under a $120-\mathrm{kV}$ operating voltage (Tecnai 12 Biotwin; FEI Co., Eindhoven, The Netherlands). Images were digitally recorded at a resolution of $1,028 \times 768$ pixels using a Megaview II (SIS Germany).

\section{Screening of Different Geographical Populations of}

Ae. aegypti for the Presence of DNV

Various populations of Ae. aegypti mosquitoes from different states of India were tested for the presence of DNV by PCR as described above. From the southern part of India, mosquitoes screened included 3 populations from the state of Kerala, 5 from Andhra Pradesh and 8 from Karnataka. The western part of India was covered by screening 11 populations from the state of Maharashtra and 2 each from Goa and Gujarat. One population each from Daman, Assam and West Bengal, which are located in eastern India, and 1 from Delhi, which represented the northern part of India, were also analyzed.

\section{Amplification, Sequencing and Phylogenetic Analysis of the $M D V$}

Viral DNA was extracted from virus stock using DNAZol (Invitrogen, Carlsbad, Calif., USA) according to the manufacturer's protocol. Overlapping primers were designed from the known sequences of AeDNV (accession No. M37899) and C. pipiens pallens DNV (CppDNV; accession No. EF579771). The complete coding region was amplified in overlapping fragments using different primer sets (table 1). The amplification was performed using Platinum Taq DNA polymerase (Invitrogen). The PCR conditions for amplification were $94^{\circ}$ for $3 \mathrm{~min}, 93^{\circ}$ for $30 \mathrm{~s}, 53^{\circ}$ for $1 \mathrm{~min}, 72^{\circ}$ for $30 \mathrm{~s}$, repeat for 35 cycles and final extension of $72^{\circ}$ for $5 \mathrm{~min}$. The amplification of the $3^{\prime}$ palin- dromic sequence was a challenge due to formation of secondary structure. To amplify this region, the DNA was denatured at $98^{\circ}$ for $10 \mathrm{~min}$ and was snap cooled on ice. Then the master mix was added to the denatured sample and was subjected to amplification. The amplicons were analyzed on $1.5 \%$ agarose gel and the amplified PCR products were gel eluted using a QIAquick Gel Extraction Kit (Qiagen, Hilden, Germany) as per the manufacturer's instructions. The purified PCR products were used for cyclic PCR using a Big Dye terminator kit (Applied Biosystems Inc., Foster City, Calif., USA). The conditions used for cyclic PCR were $96^{\circ}$ for $5 \mathrm{~min}, 96^{\circ}$ for $10 \mathrm{~s}, 50^{\circ}$ for $5 \mathrm{~s}, 60^{\circ}$ for $4 \mathrm{~s}$, repeat for 40 cycles and extension at $60^{\circ}$ for $5 \mathrm{~min}$. Cyclic PCR products were purified and products were sequenced using the ABI 3100 automated DNA sequencer.

The sequences obtained were analyzed by the Basic Local Alignment Search Tool (BLAST) and were manually curetted using the software Kodon. The curetted sequences were analyzed by GeneRunner software to determine the open reading frames (ORFs) and their properties. The curetted sequences of each individual ORF were aligned by Clustal W (as implemented in Mega 3.1), and phylogenetic analysis was performed by the neighbor joining method with 1,000 bootstrap replicates using Mega 3.1 [16]. The sequences of other MDVs available in the NCBI databank such as CppDNV [17], AeDNV [18], C6/36DNV [8] and Haemagogus equinus DNV [19] were also used in phylogenetic analysis. Sequences of DNVs isolated from other arthropods, namely hepatopancreatic parvovirus of Penaeus monodon [20], Casphalia extranea DNV [21] and Dendrolimus punctatus DNV [22], were used as out-group sequences.

\section{Results}

\section{Development of the DNV-Infected Mosquito Colony}

The DNV-infected Ae. aegypti mosquito colony was tested randomly for the presence of DNV by PCR. The colony was found to be positive for the presence of DNV. 
Fig. 1. Western blotting of the immunoprecipitated purified virus. Lane 1: pellet after $30 \%$ glycerol cushion; lane 2: supernatant after immunoprecipitation; lane 3: pellet after immunoprecipitation; lane 4: IgG included as negative control; lane 5: protein marker.

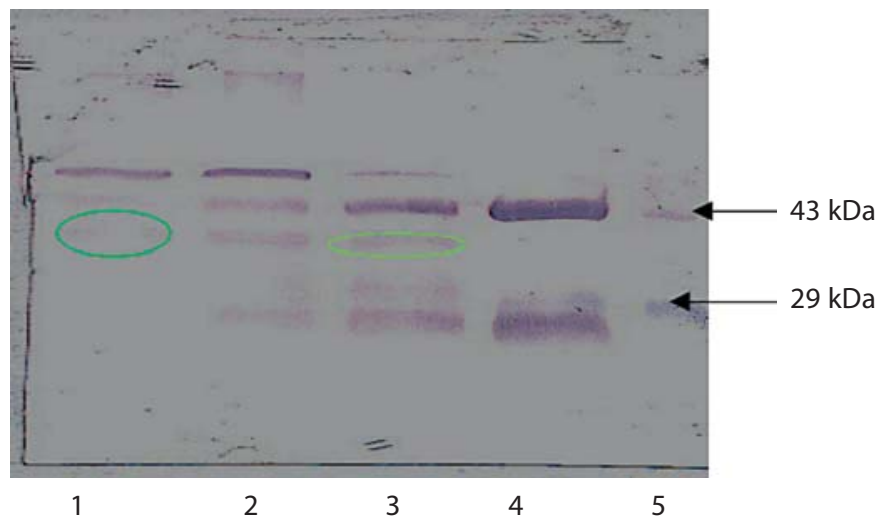

The specific band of 1,097 bases as reported for this primer pair was observed (data not shown).

\section{DNV Isolation and Purification}

All the fractions obtained from the $\mathrm{CsCl}$ gradient were found to be positive when screened by PCR, but the electron microscopy and Western blot studies showed that the $30 \%$ fraction had the highest purity and maximum concentration of DNV.

\section{SDS-PAGE and Western Blotting}

When samples collected from different fractions of the $\mathrm{CsCl}$ gradient were subjected to SDS-PAGE, it was found that the $30 \%$ fraction had bands of proteins suspected to be DNV. This fraction was used for immunoprecipitation. When the immunoprecipitated virus was subjected to SDS-PAGE and Western blotting, virus-specific bands of 40 and $41 \mathrm{kDa}$ were seen, along with IgG bands (fig. 1). This duplex of bands was similar to that reported for DNV [8].

\section{Transmisson Electron Microscopy}

When subjected to negative staining, morphologically distinct parvovirus-like particles with a size range of 18$22 \mathrm{~nm}$ could be detected at moderate to high frequency in the fields scanned (fig. 2).

\section{Presence of MDV in Different Geographical \\ Populations}

Three Ae. aegypti populations from Kerala, 2 from Andhra Pradesh and 1 from Karnataka were found to be positive for this MDV. Five populations from Maharash-

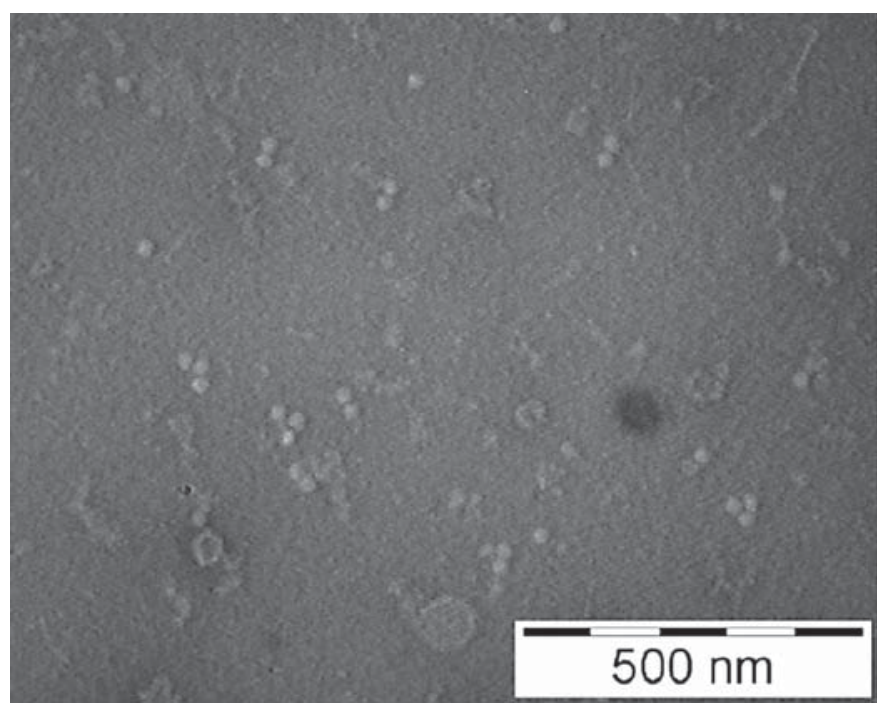

Fig. 2. Transmission electron microscopy showing icosahedral, nonenveloped particles of about $20 \mathrm{~nm}$ similar to other DNVs.

tra were positive and 1 each from Goa and Gujarat. The population from Assam was found to be positive, while those from West Bengal and Daman were found to be negative. The sample from Delhi was also positive for the MDV.

Amplification, Sequencing and Phylogenetic Analysis of the $M D V$

A total of 3,776 bases of the DNV genome were sequenced. This included 128 bases of the $3^{\prime}$ palindrome, 
Fig. 3. Phylogenetic relationship between NIVDNV and other MDVs using the left ORF. CppDNV (EF579771), AeDNV (M37899), C6/36DNV (AY095351), Haemagogus equinus DNV (HeDNV; AY605055), Dendrolimus punctatus DNV (DpDNV; NC_006555), Casphalia extranea DNV (CeDNV; NC_004288) and hepatopancreatic parvovirus of penaeid shrimp (Hpv; NC_007218) were used for Clustal $\mathrm{W}$ and phylogenetic analysis of ORFs of NIVDNV.

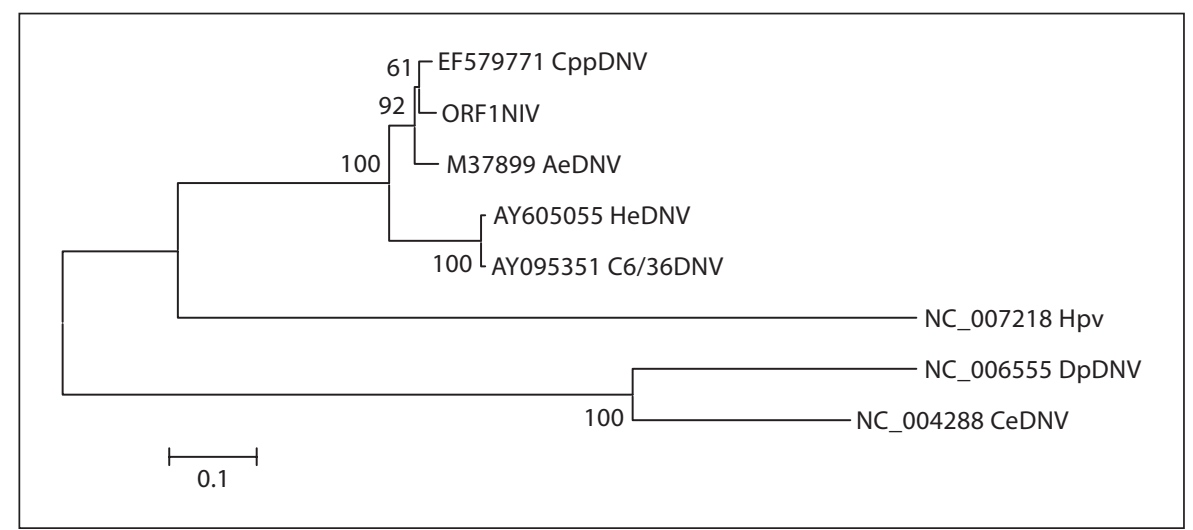

3,507 bases of coding region and 141 bases of the 5'UTR. This sequence consists of $21.2 \%$ adenine, $41.3 \%$ thymidine, $19.5 \%$ guanine and $18.0 \%$ cytosine and it encodes 3 overlapping ORFs. BLAST analysis showed 95\% similarity between this sequence and CppDNV and 93\% similarity with AeDNV. The palindromic region at the 5'UTR end could not be sequenced because of the difficulty in amplifying it.

The left and right ORFs stretch in a consecutive manner along one strand of DNA. They almost cover the entire length of the plus strand. These ORFs overlap at their extremities. Another small ORF referred to as the midORF is completely located within the left ORF, in a different reading frame. The minus-strand ORF found in AeDNV [23] was not observed here. Several putative promoter sequences were observed in the positive strand. Eight AATAAA sequences, which are signals for polyadenylation, were also found in the plus strand. They fall at positions 1020, 1443, 1566, 1976, 2214, 2391, 2906 and 3680 . The consensus promoter sequence TATAA was found at 12 sites in the genome. Sequences related to the consensus promoter sequence were also found at many places.

\section{Left ORF}

The start codon of the left ORF falls at nucleotide 129 and the ORF ends at 2676. This creates a protein of 849 amino acids (aa) with a molecular weight of approximately $97.6 \mathrm{kDa}$. This ORF has $92 \%$ amino acid homology with the NS1 protein of AeDNV. The encoded amino acid sequence also consists of a GTP/ATP binding motif with the conserved amino acid sequence GITNAGKSL. The amino acid stretch from positions 594 to 749 showed similarity with the superfamily 3 helicase of DNA viruses' domain profile according to prediction using the soft- ware Expert Protein Analysis System (Swiss Institute of Bioinformatics). A stretch of around 200 aa which is highly conserved among different DNVs was found to be located at amino acid positions 328-505.

\section{Mid ORF}

The mid ORF falls completely within the left ORF, though in a different reading frame. The putative protein of this ORF has 362 aa if the translation begins at the first AUG at nucleotide position 376 and ends at nucleotide position 1462. The protein has a molecular mass of 41.1 $\mathrm{kDa}$. This putative protein had $94 \%$ similarity with the NS2 protein of AeDNV. However, no similarity was seen with other parvoviruses.

\section{Right ORF}

This ORF begins at nucleotide position 2561 and ends at 3635. Thus, the left and right ORFs overlap at their extremities. If translation begins at the first AUG, this ORF creates a protein of 358 aa with a molecular weight of 40.6 $\mathrm{kDa}$. This protein was found to have a high degree of analogy with the viral capsid proteins of other MDVs. This ORF has $85 \%$ amino acid homology with the capsid protein of AeDNV. The putative protein contains the highly conserved sequence RGTKRKR at amino acid positions 14-20 within the glycine-rich region as reported for other MDVs. There is a glycosaminoglycan attachment site at the 27th aa, which reinforces the possibility that the right ORF may synthesize the viral capsid protein. This motif contains the consensus sequence SGAG, where the glycosaminoglycan will attach to the serine molecule.

Phylogenetic analyses using each ORF showed that this DNV isolate falls in the lineage of Brevidensovirus. A phylogenetic tree (fig. 3) was constructed for each ORF, 
Table 2. Percentage homology of the newly isolated Ae. aegypti DNV with other MDVs

\begin{tabular}{|c|c|c|c|c|c|c|}
\hline \multirow[t]{2}{*}{ Organism } & \multicolumn{2}{|c|}{ Left ORF, \% } & \multicolumn{2}{|c|}{ Mid ORF, \% } & \multicolumn{2}{|c|}{ Right ORF, \% } \\
\hline & aa & $\mathrm{nt}$ & aa & nt & aa & nt \\
\hline CppDNV & 96 & 97 & 97 & 98 & 94 & 95 \\
\hline AeDNV & 92 & 95 & 94 & 96 & 85 & 85 \\
\hline HeDNV & 67 & 81 & 74 & 81 & 77 & 69 \\
\hline C6/36DNV & 67 & 81 & 73 & 80 & 77 & 68 \\
\hline
\end{tabular}

but as those trees showed similar topology, only the NS1 gene (left ORF) is presented here. This virus shows maximum homology with CppDNV. The percentage homology of nucleotides and amino acids for each ORF (left, mid and right) was compared with known MDVs and is presented in table 2 .

\section{Discussion}

This is the first report of the isolation and characterization of MDV from India. The present study shows infection in various geographical populations of Ae. aegypti mosquitoes from different states of India, indicating that MDV is widely distributed. It has been observed by earlier workers that mosquito strains may differ in their susceptibility and response to DNV infections [24]. Other studies have shown seasonal and spatial variation in natural DNV infection in Anopheles mosquitoes [6].

AeDNV isolated from Ae. aegypti mosquitoes showed only 2 proteins of 38 and $40 \mathrm{kDa}$ [18]. Similarly, in our study, immunoprecipitation and SDS-PAGE in conjunction with Western blot studies showed the presence of 2 proteins of 40 and $41 \mathrm{kDa}$. It is well known that insect DNVs are small, 25- to 28-nm-diameter, nonenveloped, icosahedral viruses. These features could be visualized on an electron microscope and hence, further quick characterization became possible. After confirmation of DNV by SDS-PAGE, Western blotting and partial PCR amplification, attempts were made to amplify the complete genome of the virus, but due to a palindrome sequence, only the 3'UTR, the complete coding region and part of the $5^{\prime}$ UTR were sequenced.

The sequencing data confirmed that this virus encodes 3,507 bases of coding region and 3 ORFs, as reported earlier. ORF1 (left ORF) is 2,547 nt long and encodes a nonstructural protein (NS1) of 849 aa. ORF2 (mid
ORF) is 1,086 nt and encodes the NS2 protein, which is 358 aa long. ORF3 (right ORF) is 1,074 nt long and encodes 362 aa for a capsid protein (VP). The genome organization is very similar to the genome of AeDNV. The minus-strand ORF found in AeDNV [18] was not observed here. The right ORF of our virus has $6 \mathrm{~N}$ glycosylation sites, while AeDNV has 5 [25]. VP of the new isolate contains the highly conserved sequence RGTKRKR as reported for other DNVs from mosquitoes at a similar amino acid position [14-20] within the glycine-rich region.

Phylogenetic analyses performed for all 3 ORFs showed that the isolated DNV falls in the lineage of MDV. Our analysis suggests separation of AeDNV and AalDNV based on all 3 ORFs and that NIVDNV is closer to AeDNV than the AalDNV genome. This virus shows maximum homology with CppDNV of 96, 97 and 94\% similarity for the left, mid and right ORFs, respectively, at the amino acid level. The genome similarity of this virus with CppDNV, which was isolated in China [17], indicates the possibility that this virus evolved during a similar time period in this continent and can infect different species of mosquitoes. Widespread distribution of $\mathrm{DNV}$ in Ae. aegypti, the principal vector of dengue and chikungunya in this country, calls for in-depth studies on how the presence of this virus affects the vector competence of mosquitoes.

\section{Acknowledgments}

Aruna Sivaram thanks the Department of Biotechnology, India, for providing a research fellowship and Dr. A.C. Mishra, Director, National Institute of Virology, for critical comments on the manuscript.

References

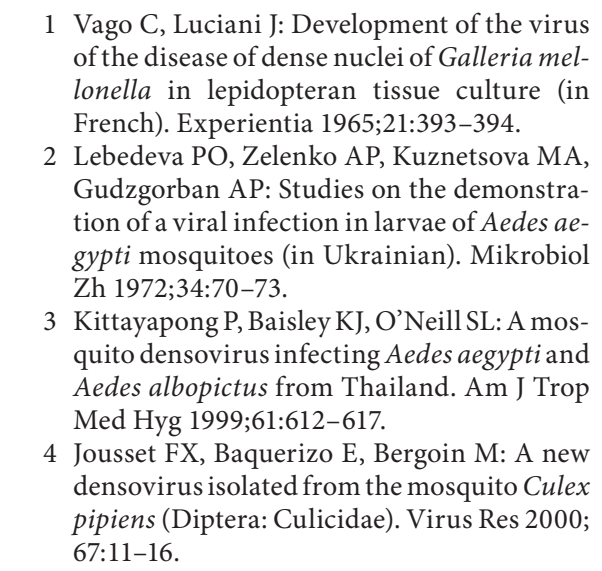

Sivaram/Barde/Kumar/Yadav/Gokhale/ Basu/Mourya 
5 Pattanakitsakul SN, Boonnak K, Auethavornanan K, Jairungsri A, Duangjinda T, Puttatesk P, Thongrungkiat S, Malasit P: A new densovirus isolated from the mosquito Toxorhynchites splendens (Wiedemann) (Diptera: Culicidae). Southeast Asian J Trop Med Public Health 2007;38:283-293.

-6 Rwegoshora RT, Baisley KJ, Kittayapong P: Seasonal and spatial variation in natural densovirus infection in Anopheles minimus s.l. in Thailand. Southeast Asian J Trop Med Public Health 2000;31:3-9.

$\checkmark 7$ Jousset FX, Barreau C, Boublik Y, Cornet M: A parvo-like virus persistently infecting a C6/36 clone of Aedes albopictus mosquito cell line and pathogenic for Aedes aegypti larvae. Virus Res 1993;29:99-114.

$\checkmark 8$ Chen S, Cheng L, Zhang Q, Lin W, Lu X, Brannan J, Zhou ZH, Zhang J: Genetic, biochemical, and structural characterization of a new densovirus isolated from a chronically infected Aedes albopictus C6/36 cell line. Virology 2004;318:123-133.

9 O’Neill SL, Kittayapong P, Braig HR, Andreadis TG, Gonzalez JP, Tesh RB: Insect densoviruses may be widespread in mosquito cell lines. J Gen Virol 1995;76:2067-2074.

10 Ward TW, Jenkins MS, Afanasiev BN, Edwards M, Duda BA, Suchman E, JacobsLorena M, Beaty BJ, Carlson JO: Aedes aegypti transducing densovirus pathogenesis and expression in Aedes aegypti and Anopheles gambiae larvae. Insect Mol Biol 2001;10: 397-406.
-11 Barreau C, Jousset FX, Bergoin M: Pathogenicity of the Aedes albopictus parvovirus (AaPV), a denso-like virus, for Aedes aegypti mosquitoes. J Invertebr Pathol 1996;68: 299-309.

12 Barreau C, Jousset FX, Bergoin M: Venereal and vertical transmission of the Aedes albopictus parvovirus in Aedes aegypti mosquitoes. Am J Trop Med Hyg 1997;57:126-131.

13 Burivong P, Pattanakitsakul SN, Thongrungkiat S, Malasit P, Flegel TW: Markedly reduced severity of Dengue virus infection in mosquito cell cultures persistently infected with Aedes albopictus densovirus (AalD NV). Virology 2004;329:261-269.

14 Wei W, Shao D, Huang X, Li J, Chen H, Zhang Q, Zhang J: The pathogenicity of mosquito DNV (C6/36DNV) and its interaction with dengue virus type II in Aedes albopictus. Am J Trop Med Hyg 2006;5:1118-1126.

15 Ohsawa K, Ebata N: Silver staining for detecting 10-femtogram quantities of protein after polyacrylamide gel electrophoresis. Anal Biochem 1983;135:409-415.

16 Kumar S, Tamura K, Nei M: MEGA3: integrated software for Molecular Evolutionary Genetics Analysis and sequence alignment. Brief Bioinform 2004;5:150-163.

17 Zhai YG, Lv XJ, Sun XH, Fu SH, Gong ZD, Fen Y, Tong SX, Wang ZX, Tang Q, Attoui H, Liang GD: Isolation and characterization of the full coding sequence of a novel densovirus from the mosquito Culex pipiens pallens. J Gen Virol 2008;89:195-199.

-18 Afanasiev BN, Galyov EE, Buchatsky LP, Kozlov YV: Nucleotide sequence and genomic organization of Aedes densonucleosis virus. Virology 1991;185:323-336.
19 Paterson A, Robinson E, Suchman E, Afanasiev B, Carlson J: Mosquito densonucleosis viruses cause dramatically different infection phenotypes in the C6/36 Aedes albopictus cell line. Virology 2005;337:253261.

20 Sukhumsirichart W, Attasart P, Boonsaeng V, Panyim S: Complete nucleotide sequence and genomic organization of hepatopancreatic parvovirus (HPV) of Penaeus monodon. Virology 2006;346:266-277.

21 Fediere G, Li Y, Zadori Z, Szelei J, Tijssen P: Genome organization of Casphalia extranea densovirus, a new iteravirus. Virology 2002; 292:299-308.

22 Wang J, Zhang J, Jiang H, Liu C, Yi F, Hu Y: Nucleotide sequence and genomic organization of a newly isolated densovirus infecting Dendrolimus punctatus. J Gen Virol 2005;86: 2169-2173.

23 Afanasiev BN, Kozlov YV, Carlson JO, Beaty BJ: Densovirus of Aedes aegypti as an expression vector in mosquito cells. Exp Parasitol 1994;79:322-339.

24 Ledermann JP, Suchman EL, Black WC, Carlson JO: Infection and pathogenicity of the MDVs AeDNV, HeDNV, and APeDNV in Aedes aegypti mosquitoes (Diptera: Culicidae). J Econ Entomol 2004;97:18281835.

25 Boublik Y, Jousset FX, Bergoin M: Complete nucleotide sequence and genomic organization of the Aedes albopictus parvovirus (AaPV) pathogenic for Aedes aegypti larvae. Virology 1994;200:752-763. 